\title{
Nighttime $D$-region equivalent electron density determined from tweek sferics observed in the South Pacific Region
}

\author{
Sushil Kumar' ${ }^{1}$, Anil Deo², and V. Ramachandran ${ }^{1}$ \\ ${ }^{1}$ School of Engineering and Physics, The University of the South Pacific, Suva, Fiji \\ ${ }^{2}$ School of Applied Science, Fiji Institute of Technology, Suva, Fiji
}

(Received November 5, 2008; Revised March 2, 2009; Accepted March 2, 2009; Online published August 31, 2009)

\begin{abstract}
Atmospherics or sferics that originate from lightning discharges on propagating large distances in the Earthionosphere waveguide, particularly at the night, form dispersed sferics called tweeks. Tweeks are novel diagnostic tool to monitor the nighttime $D$-region ionosphere. Mean equivalent electron density $n_{\mathrm{em}}$ at mean tweek reflection heights $h_{\mathrm{m}}$ and electron density profile have been estimated using the higher harmonic tweeks recorded in the time between $21-03 \mathrm{hrs}$ LT at Suva $\left(18.2^{\circ} \mathrm{S}, 178.3^{\circ} \mathrm{E}\right)$, Fiji, during a period March-December 2006. The values of $n_{\mathrm{em}}$ vary from $29-170 \mathrm{~cm}^{-3}$ in the altitude range of about $3.5 \mathrm{~km}$ at $h_{\mathrm{m}}$ of about $83 \mathrm{~km}$. In terms of usually used exponential electron density profile, the ionospheric reference height and the exponential sharpness factor are calculated to be $83.1 \mathrm{~km}$ and $0.64 \mathrm{~km}^{-1}$, respectively. The scale height is calculated to be $1.9 \mathrm{~km}$. Equivalent electron density profile of the nighttime lower ionosphere, using tweek method, shows lower values of electron density by about $20-45 \%$ than those obtained from the IRI-2001 model.
\end{abstract}

Key words: Earth-ionosphere waveguide, tweek atmospherics, reflection heights, equivalent electron densities.

\section{Introduction}

The $D$-region of ionosphere ranges from $60-90 \mathrm{~km}$ wherein collisions between charged particles and neutrals dominate. The lower ionosphere, consisting of $D$ and $E$ regions, is too high for balloons and too low for the satellite measurements. Radio sounding does not work, particularly at night, since electron densities in this region are low to reflect high frequency radio waves with ionosondes and incoherent radars. Therefore, the lower ionosphere $(\sim 60$ $140 \mathrm{~km}$ ) remains the least studied region of the Earth's atmosphere (Cummer and Inan, 2000). Impulse-like signals containing bulk of the energy in the Extremely Low Frequency (ELF) and the Very Low Frequency (VLF) bands generated by lightning discharges propagate through the Earth-ionosphere waveguide (EIWG) by multiple reflections and are received as radio atmospherics or sferics at receiver. Dispersed sferics called tweeks due to long distance propagation in the EIWG, particularly at night, when attenuation is less, contain information about the state of the lower ionosphere along the propagation path (Hayakawa et al., 1994; Cummer et al., 1998; Ohya et al., 2006, 2008; Ferencz et al., 2007).

In this work, tweeks with higher harmonics observed during the period March-December 2006 at the low latitude station, Suva (geog. lat. $18.2^{\circ} \mathrm{S}$, geog. long. $178.3^{\circ} \mathrm{E}$ ), Fiji, are utilized to estimate the nightime $D$-region equivalent electron density at the tweek reflections heights and the equivalent electron density profile of the nighttime lower ionosphere. The broadband ELF-VLF data were recorded

Copyright (c) The Society of Geomagnetism and Earth, Planetary and Space Sciences (SGEPSS); The Seismological Society of Japan; The Volcanological Society of Japan; The Geodetic Society of Japan; The Japanese Society for Planetary Sciences; TERRAPUB. using World Wide Lightning Location Network (WWLLN) VLF setup at The University of the South Pacific, Suva, Fiji. There are 28 universities and national institutes all over the world currently participating in WWLLN.

\section{Theoretical Background}

The electromagnetic field in the waveguide can be decomposed into a sequence of independent field structures (modes) which propagate with different group velocities. Each of the modes $(n)$ is defined by its cutoff frequency $f_{\text {cn }}$ which is determined by the height $h$ of the waveguide or the VLF reflection height. We consider a conventional spherical EIWG and suppose the Earth as an ideal perfect conductor. We consider that the lower ionosphere (upper boundary of EIWG) also acts as an ideal perfect conductor but at higher altitudes for higher harmonics of the same tweeks. For a waveguide having perfectly conducting boundaries, the reflection heights of different harmonics is given by (Budden, 1961; Yamashita, 1978):

$$
h=\frac{n c}{2 f_{\mathrm{cn}}}
$$

where $c$ is the velocity of light in free space.

The distribution of charged particles in the ionosphere depends in a complicated way on latitude, solar zenith angle, season, and solar activity etc. In the simplest approach, the exponential increase in the lower-ionospheric electron density $n_{\mathrm{e}}$ expressed in $\mathrm{cm}^{-3}$, can be described by Wait profile, valid up to about $100 \mathrm{~km}$ altitude (Wait and Spies, 1964):

$$
\begin{aligned}
n_{\mathrm{e}}(h)= & 1.43 \times 10^{7} \exp \left(-0.15 h^{\prime}\right) \\
& \times \exp \left[(\beta-0.15)\left(h-h^{\prime}\right)\right], \quad \mathrm{cm}^{-3}
\end{aligned}
$$


where $h^{\prime}$ is the ionospheric reference height in $\mathrm{km}$ and $\beta$ is the parameter measured in $\mathrm{km}^{-1}$ that describes the sharpness of the electron density profile.

The propagation of electromagnetic waves in collisional magnetized plasma is formulated by the magnetoionic theory which also applies to the ionosphere (Ratcliffe, 1959). Ionosphere is weakly ionized, inhomogeneous, collisional, and anisotropic plasma. The complex refractive index $\mu$ of waves in the ionosphere is given by the famous AppletonHartree equation (Budden, 1961):

$$
\mu^{2}=1-\frac{X}{1-i Z-\left[\frac{Y_{\mathrm{T}}^{2}}{2(1-X-i Z)}\right] \pm\left[\sqrt{\frac{Y_{\mathrm{T}}^{4}}{4(1-X-i Z)^{2}}+Y_{\mathrm{L}}^{2}}\right]},
$$

where $X, Y_{\mathrm{T}}, Y_{\mathrm{L}}$ and $Z$ are dimensionless magneto-ionic parameters, defined as:

$$
\begin{aligned}
X & =\left(\frac{\omega_{\mathrm{p}}}{\omega}\right)^{2}, Y_{\mathrm{T}}=\frac{\omega_{\mathrm{H}}}{\omega} \sin \theta=Y \sin \theta, \\
Y_{\mathrm{L}} & =\frac{\omega_{\mathrm{H}}}{\omega} \cos \theta=Y \cos \theta, \text { and } Z=\frac{\nu_{\mathrm{e}}}{\omega},
\end{aligned}
$$

where $\omega_{\mathrm{p}}, \omega_{\mathrm{H}}$, and $\omega$ are the angular plasma frequency, electron gyrofrequency, and wave frequency, respectively. $\nu_{\mathrm{e}}$ is the angular electron-neutral collision frequency and $\theta$ is the angle between the propagation direction and the Earth's magnetic field vector. $\omega_{\mathrm{p}}$ is given by: $\omega_{\mathrm{p}}=\sqrt{n_{\mathrm{e}} e^{2} / \epsilon_{0} m_{\mathrm{e}}}$, where $n_{\mathrm{e}}$ is the electron density, $e\left(=-1.602 \times 10^{-19} \mathrm{C}\right)$ is charge of an electron, $\epsilon_{0}\left(=8.854 \times 10^{-12} \mathrm{~F} / \mathrm{m}\right)$ is permittivity of free space, and $m_{\mathrm{e}}\left(=9.109 \times 10^{-31} \mathrm{~kg}\right)$ is the mass of an electron.

The $v_{\mathrm{e}}$ profile can be very closely approximated by (Wait and Spies, 1964):

$$
v_{\mathrm{e}}=1.816 \times 10^{11} \exp (-0.15 h), \mathrm{s}^{-1}
$$

where $h$ is the altitude measured in $\mathrm{km}$.

VLF waves reflect at the bottom-side of the ionosphere, that is, in the $D$-region. The upper sign "+" in Eq. (3) corresponds to ordinary-mode (O-mode) waves and lower sign "_" corresponds to extraordinary-mode (X-mode) waves. $\mathrm{X}$-mode waves undergo full reflection at the altitude at which $\mu$ becomes zero. The O-mode waves are not reflected and penetrate into the ionosphere. The condition of full wave reflection for X-mode wave can be easily obtained using the quasi-longitudinal (QL) approximation $(\theta$ small). Under QL approximation, excluding the terms containing $\sin ^{2} \theta$ and $\sin ^{4} \theta$ and equating $Y_{\mathrm{L}}$ to $Y$, Eq. (3) for VLF waves becomes:

$$
\mu^{2}=1-\frac{X}{1-i Z \pm Y} .
$$

At VLF, both $\omega_{\mathrm{H}}$ and $\nu_{\mathrm{e}}$ in the lower ionosphere at the VLF reflection heights are larger than the tweek wave frequency $\left(v_{\mathrm{e}}>\omega\right.$ and $\omega_{\mathrm{H}} \gg \omega$ i.e. $\left.Y>1\right)$. The value of $X$ where $\mu^{2}$ becomes zero is given by following condition:

$$
X=1-i Z \pm Y \text {. }
$$

The $\mathrm{X}$-mode waves would correspond to $X=1-i Z+Y$ when $Y>1$ and $X=1-i Z-Y$ when $Y<1$. For tweek frequencies (ELF/VLF) of our interest, the condition of reflection for $\mathrm{X}$-mode waves would be met only with $X=1-i Z+Y$. Therefore, the values of $n_{\mathrm{e}}$ at the reflection heights of tweeks are obtained from $X=1-i Z+Y$. Thus the magnitude of $X$ is given as:

$$
X=\left[(1+Y)^{2}+Z^{2}\right]^{\frac{1}{2}}
$$

Substituting the values of $X, Y$ and $Z$, expression for $n_{\mathrm{e}}$ at $h$ of different harmonics of tweeks can be obtained as:

$$
\begin{aligned}
n_{\mathrm{e}}= & 1.9790 \times 10^{-9} \times f_{\mathrm{cn}} \times\left\lfloor\left\{2 \pi\left(f_{\mathrm{cn}}+f_{\mathrm{H}}\right)\right\}^{2}\right. \\
& \left.+\left(1.816 \times 10^{11} \exp (-0.15 \times h)\right)^{2}\right\rfloor^{\frac{1}{2}}, \mathrm{~cm}^{-3}
\end{aligned}
$$

where $f_{\mathrm{H}}$ is the electron gyro frequency. Second term is the contribution to $n_{\mathrm{e}}$ due to electron-neutral collisional frequency and is very small as compared to the contribution due to first term. In the absence of the last term the expression reduces to that given by Ohya et al. (2003).

\section{Observational Data}

WWLLN system used for ELF-VLF consists of a short $(1.5 \mathrm{~m})$ whip antenna, pre-amplifier, and a receiving unit. Whip antenna receives the vertical electric field component $E_{Z}$ of transverse magnetic (TM) and quasi-transverse electromagnetic (QTEM) mode waves. Global Position System (GPS) is utilized for precise measurements of arrival times of tweeks. The details of the system have been described by Dowden et al. (2002). The lightning software installed in WWLLN PC records the broadband data in files of $11 \mathrm{MB}$ per minute. Data were recorded for 5 minutes at every hour in the nighttime during March-December 2006. The files were analyzed using a MATLAB code which produces spectrograms (frequency $f$ vs. time $t$ ) of one-second duration. To accurately read the frequency and corresponding arrival times, we have used Getdata Graph Digitizer software. First the spectrograms were loaded into paint software and saved in bitmap format. In the next step, these bitmap files were uploaded in the GetData Graph Digitizer software and calibrated for the frequency and time axes of the spectrograms. The cutoff frequency $f_{\mathrm{c} 1}$ of first harmonics and two nearby frequencies $\left(f_{1}\right.$ and $\left.f_{2}\right)$ together with their corresponding time $\left(t_{1}\right.$ and $\left.t_{2}\right)$ displayed by GetData Graph Digitizer software were then obtained. The propagation paths of tweeks observed at Suva are mostly over the sea and we have considered tweeks which propagated about $6000 \mathrm{~km}$, thus the overall attenuation is small especially for the first harmonic frequency components. Therefore, we consider that the energy of waves at $f_{\mathrm{c} 1}$ does not go below the noise level and $f_{\mathrm{c} 1}$ is measured quite accurately using the GetData Graph Digitizer which can show the frequency components above the noise level clearly. Whereas, higher harmonics of tweeks while propagating in the EIWG suffer higher attenuation as compared to the first harmonic which is progressively higher for higher harmonic waves and increases sharply as frequencies approach the cutoff frequency (Kumar el al., 2008). Due to this, some of the frequency components of higher harmonics of tweeks near their cutoff frequencies might not be received. The accurate cutoff frequencies of higher harmonics were obtained using 

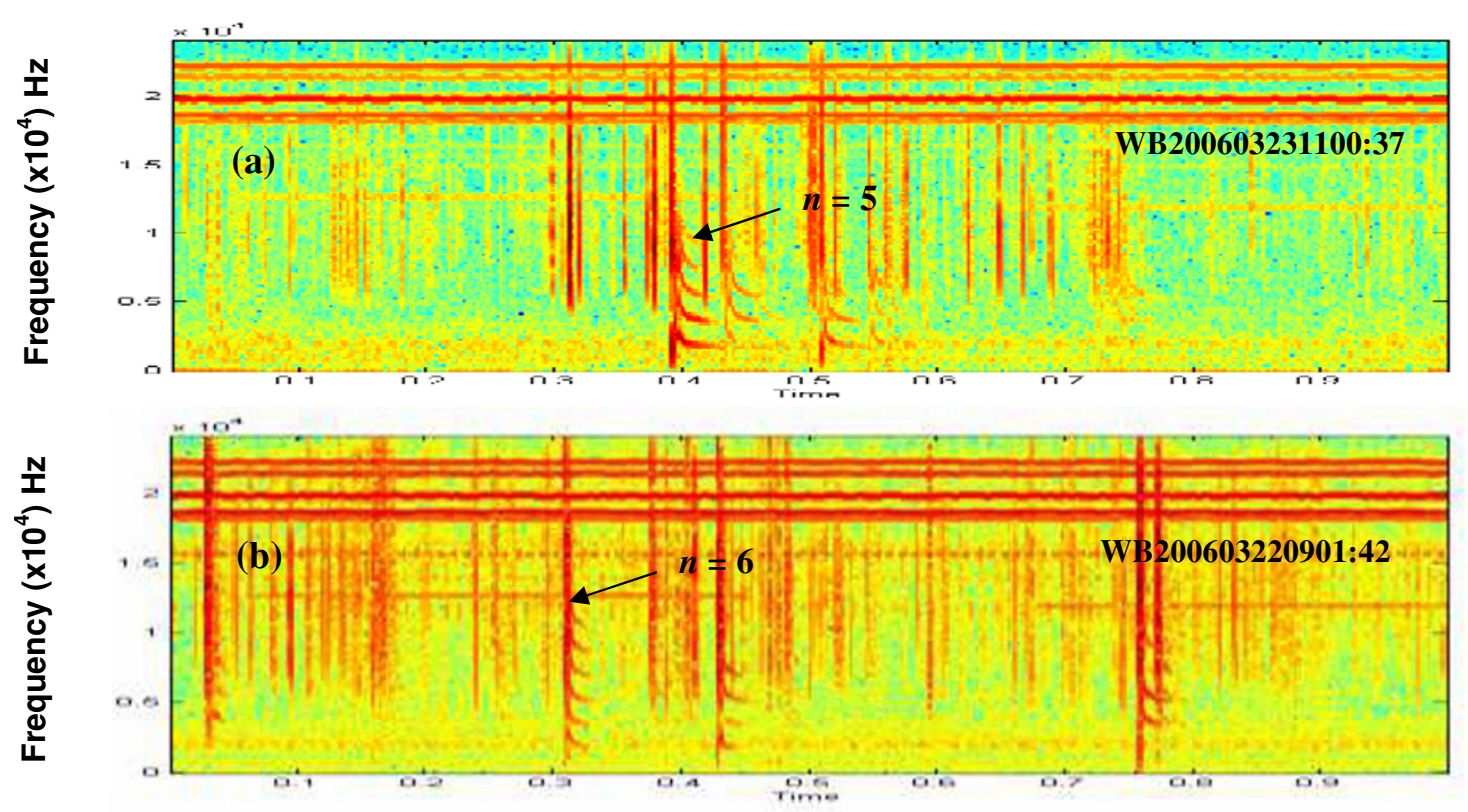

Fig. 1. Typical spectrograms showing tweeks observed on (a) 23 March 2006 at 23:00:37 hrs LT, and (b) 22 March 2006 at 21:01:42 hrs LT.

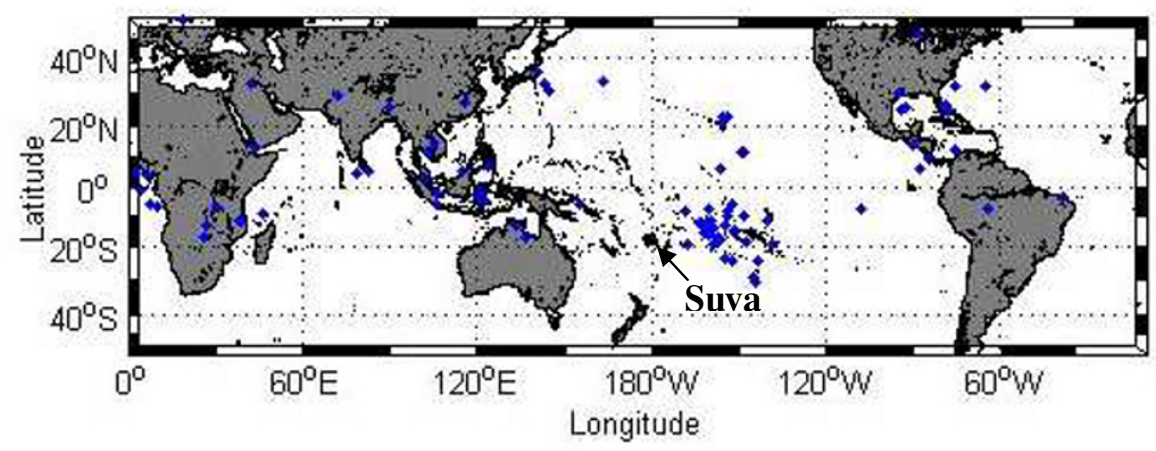

Fig. 2. Lightning locations of tweeks determined from WWLLN data. Blue color square marks indicate location of lightnings associated with tweeks.

the above steps and by fitting (extrapolating) the dispersion up to the time of arrival of the cutoff frequency of the first harmonic of tweeks. The resolution in the frequency and time components is $35 \mathrm{~Hz}$ and $1 \mathrm{~ms}$, respectively.

\section{Results and Discussion}

\subsection{General overview of tweeks observed at Suva}

First observations of tweeks at this station were made during September 2003-July 2004 for a total of $30 \mathrm{~min}$. at different hours in the night and the day times. Data analysis showed no tweeks in the daytime recordings. General features of tweeks recorded during above period have been published by Kumar et al. (2008). A more systematic recoding of data was made during March-December 2006 when ELF-VLF data were recorded for 5 minutes at every hour in the nighttime (18-06 hrs LT). A total of 1039 tweeks were selected on the basis of clarity of tweeks seen in the spectrograms. Figure 1(a,b) presents the spectrograms showing typical multimode tweeks observed on (a) 23 March 2006 at 23:00:37 hrs LT and (b) 22 March 2006 at 21:01:42 hrs
LT. The frequency components below the cutoff frequency of first mode (harmonic) correspond to zeroth-order mode $\left(\mathrm{TM}_{0}\right)$. To identify the location of lightnings associated with tweeks, WWLLN data were analyzed. The lightning stroke detection within $50 \mathrm{~ms}$ earlier to tweeks observation and that best matched the tweek propagation distance (in case of more than one lightning within $50 \mathrm{~ms}$ ) was taken as the causative lightning of that particular tweek. Under these criteria out of 1039 tweeks, the lightning source locations of only 87 tweeks were found. This is due to the low detection efficiency of WWLLN system. WWLLN detects global lightnings with return stroke currents of more than $\sim 50 \mathrm{kA}$ with spatial and temporal accuracy of roughly 10-20 km and $10 \mu \mathrm{s}$, respectively, and has global detection efficiency less than 4\% (Rodger et al., 2006), although a much higher detection efficiency for stronger lightnings is expected. Figure 2 shows the locations of lightnings associated with 87 tweeks. The causative lightnings were distributed over a wide range of locations but majority of them $(\sim 60 \%)$ occurred in the geographic latitude and longitude ranges of 


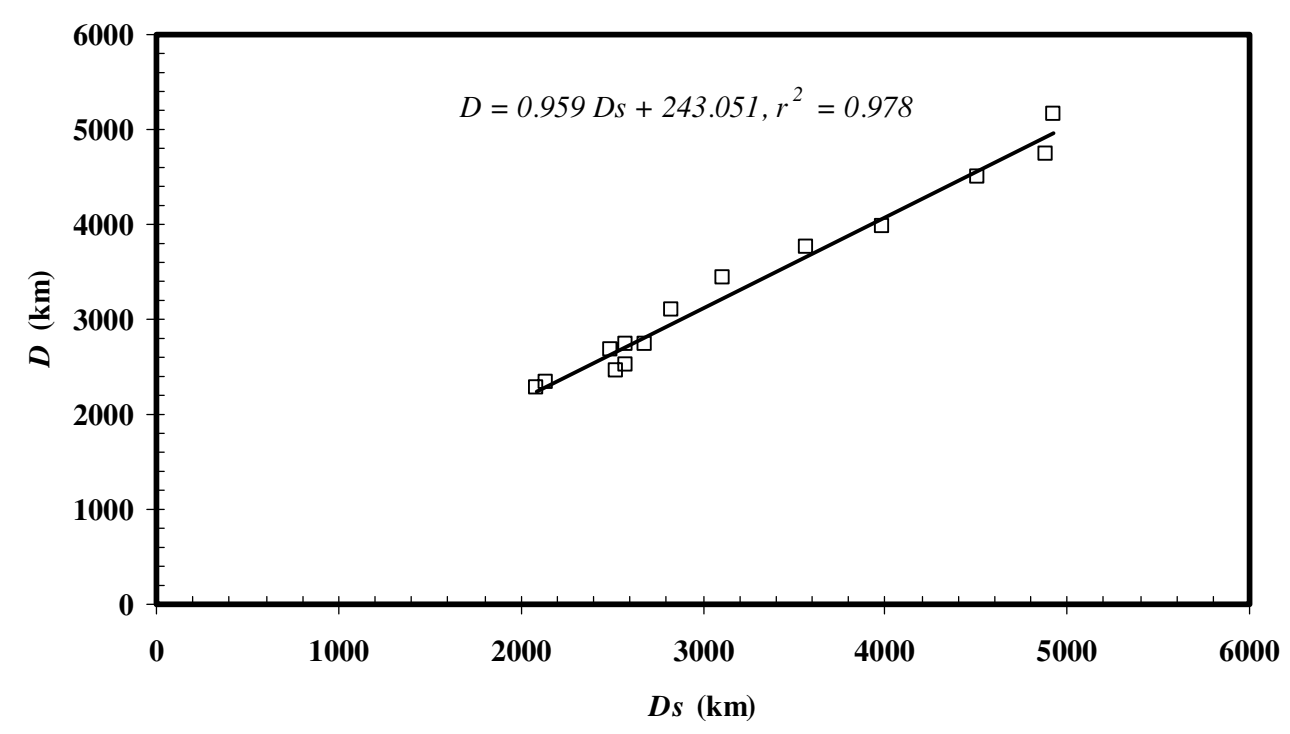

Fig. 3. Correlation of great circle distance $\left(D_{\mathrm{s}}\right)$ with propagation distance $(D)$ calculated using Haversine and tweek methods, respectively.

Table 1. Reflection heights $h$ and electron densities $n_{\mathrm{e}}$ at $h$ obtained from tweeks shown in Fig. 1.

\begin{tabular}{cccccc}
\hline Spectrogram & $\begin{array}{c}\text { Time } \\
(\text { LT hrs })\end{array}$ & $\begin{array}{c}\text { Mode number } \\
(n)\end{array}$ & $\begin{array}{c}\text { Cut-off frequency } \\
f_{\mathrm{c}}(\mathrm{kHz})\end{array}$ & $\begin{array}{c}\text { Tweek reflection } \\
\text { height } h(\mathrm{~km})\end{array}$ & $\begin{array}{c}\text { Electron density } \\
n_{\mathrm{e}}\left(\mathrm{el} . / \mathrm{cm}^{3}\right)\end{array}$ \\
\hline \hline $\mathrm{a}$ & $23: 00: 37.391$ & 1 & 1.794 & 83.6 & 29.1 \\
& 2 & 3.588 & 83.6 & 58.3 \\
& 3 & 5.323 & 84.5 & 86.6 \\
& 4 & 7.117 & 84.3 & 115.9 \\
& & 8.793 & 85.3 & 143.3 \\
\hline $\mathrm{b}$ & $22: 01: 42.308$ & 1 & 1.814 & 82.6 & 29.6 \\
& & 2 & 3.518 & 85.3 & 57.1 \\
& & 3 & 5.263 & 85.5 & 113.8 \\
& 4 & 6.997 & 85.8 & 143.3 \\
& & 8 & 8.792 & 85.3 & 170.5 \\
\hline
\end{tabular}

$10^{\circ} \mathrm{S}$ to $30^{\circ} \mathrm{S}$ and $140^{\circ} \mathrm{W}$ to $170^{\circ} \mathrm{W}$, respectively, which are geographic locations of a group of islands. Out of 1039 tweeks observed in the nighttime (18-06 hrs LT), tweeks observed during 21-03 hrs LT and those propagated distances up to 5000 to as high as $5900 \mathrm{~km}$, were selected, so that the error contribution due to day-time propagation path particularly for tweeks coming from E-W directions and the refraction on the day-night terminator is avoided. Under these criteria we end up with 448 tweeks which are used for analysis in this paper. Tweeks propagation distance $(D)$ in the spherical EIWG having perfectly conducting boundaries were calculated using the method used by Kumar et al. (2008), given as $D=\delta t\left(v_{\mathrm{g} f_{1}} \times v_{\mathrm{g} f_{2}}\right)\left|v_{\mathrm{g} f_{1}}-v_{\mathrm{g} f_{2}}\right|^{-1}$, where $\delta t=\left|t_{1}-t_{2}\right|$ is the difference in arrival times of two frequencies $f_{1}$ and $f_{2}$ close to the cutoff frequency of the first harmonic of tweeks and $v_{\mathrm{g} f_{1}}$ and $v_{\mathrm{g} f_{2}}$ are the corresponding group velocities. The distances calculated by this method are within the accuracy of about $\pm 900 \mathrm{~km}$. To check the validity of tweek method of calculating the propagation distance, the great circle distance $\left(D_{\mathrm{s}}\right)$ between the identified locations of lightning and the measurement site were calculated using Haversine formula (online at http://www.movable-type.co.uk/scripts/LatLong.html), for
14 best matched lightning locations. Plot of $D_{\mathrm{s}}$ against $D$ is shown in Fig. 3. The squared value of linear correlation coefficient $r^{2}$ for the graph is 0.98 indicating a strong correlation between the propagation distances calculated both by Haversine and tweek methods.

\subsection{Equivalent electron density at reflections heights}

At ELF and VLF, the Earth's surface and the lower ionosphere act as good electrical conductors. For tweeks considered in this paper, most of their propagation is on the sea so that the Earth can be considered as perfect conductor, but it is essential to treat the ionosphere as an anisotropic medium whose conductivity increases exponentially with height. We consider that the ionospheric conductivity does not vary with latitude and longitude along the propagation paths of tweeks. The normal reflection height of the electromagnetic waves in the ionosphere varies with frequency, with lower frequencies usually reflected at lower heights due to lower electron densities (Cummer and Inan, 2000). The fact that ELF and VLF waves are reflected by the $D$ region and $D$-region is a significantly better reflector at the night than at the day makes them useful diagnostic tools to estimate the nighttime electron densities $n_{\mathrm{e}}$ at the ionospheric reflection heights $h$. These (partial) reflections oc- 


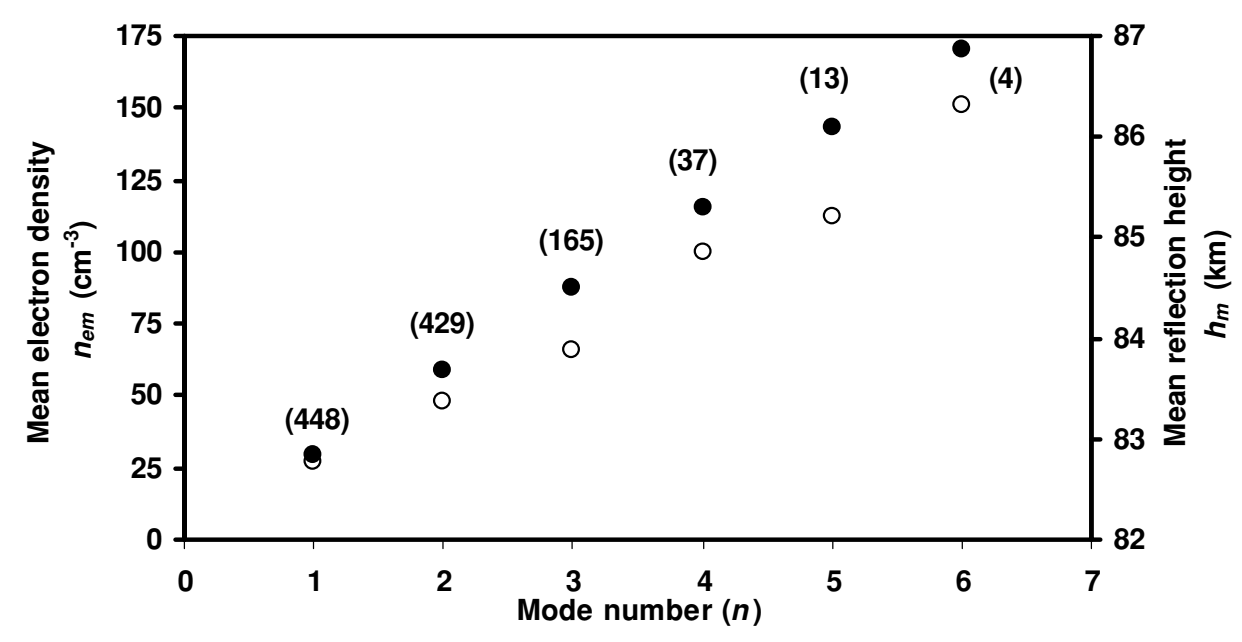

Fig. 4. Mean electron densities $n_{\mathrm{em}}$ (left hand scale, solid circles) at mean reflection heights $h_{\mathrm{m}}$ (right hand scale, hollow circles) calculated using cut-off frequency of different modes $n$ for 448 tweeks observed during March-December 2006. The numbers in the parentheses indicate the total number of tweeks utilized.

cur because the electron density in the lower ionosphere increases rapidly and higher harmonics are reflected from the higher altitudes. Using a qualitative analysis of radio wave propagation in infinite, collisionless, anisotropic, weakly ionized ionospheric plasma and by analyzing tweeks up to the 8th harmonics observed during January-April 1991, Shvets and Hayakawa (1998) estimated the numerical value of equivalent $n_{\mathrm{e}}$ at $h$ of tweeks. In this paper, the values of $h$ and $n_{\mathrm{e}}$ have been calculated using Eqs. (1) and (8), respectively, for collisional ionosphere, using the modes $n=$ 1-6 of tweeks shown in Fig. 1, and are presented in Table 1. Calculations have been made using $f_{\mathrm{H}}=1.3 \mathrm{MHz}$ for the latitudes and altitude range of our interest. It can be noted from Table 1 that $n_{\mathrm{e}}$ varies from $30-171 \mathrm{~cm}^{-3}$ in the altitude range about $3.5 \mathrm{~km}$ at the height of about $82.6 \mathrm{~km}$. The calculated value of $n_{\mathrm{e}}$ using the International Reference Ionosphere (IRI-2001) model at a height $82.6 \mathrm{~km}$, on 22 March 2006 at 21:00 hrs LT, the time of observation of tweeks marked in panels (a) and (b) of Fig. 1, is $85 \mathrm{~cm}^{-3}$ which is higher compared to the values shown in Table 1.

Under the selection criteria described in Section 4.1, a total of 448 tweeks were selected for the analysis from the broadband data recorded during the period MarchDecember 2006. The mean cutoff frequency $f_{\mathrm{cm}}$ of different modes and mean fundamental frequency $f_{\mathrm{cm}} / n$ with mode number $n$ were obtained from these tweeks. The $f_{\mathrm{cm}} / n$ changes from $1.812 \mathrm{kHz}$ to $1.738 \mathrm{kHz}$ for $n=1-6$ which indicates that the cut-off frequencies of higher harmonics $(n>1)$ are not exact multiples of the cut-off frequency of first mode $f_{\mathrm{c} 1}$ and $n$, rather are slightly lower than $f_{\mathrm{c} 1} \times n$. Similar variation of $f_{\mathrm{cm}} / n$ for 503 tweeks recorded at this station in the nighttime (18-06 hrs LT) during September 2003-July 2004 was reported by Kumar et al. (2008).

The $f_{\mathrm{cm}} / n$ and $f_{\mathrm{cm}}$ are used to calculate mean reflection height $h_{\mathrm{m}}$ and mean electron densities $n_{\mathrm{em}}$ from different harmonics (1-6) using Eqs. (1) and (8), respectively. The $n_{\mathrm{em}}$ at $h_{\mathrm{m}}$ for each mode is plotted in Fig. 4 . The numbers in the parentheses indicate the total number of tweeks used in the analysis. It is found that the $n_{\mathrm{e}}$ increases from 29.4-
$170.0 \mathrm{~cm}^{-3}$ over the $h_{\mathrm{m}}$ in the range of $82.8-86.3 \mathrm{~km}$, for $n=1-6$. The frequency components from the spectrograms are measured with an accuracy of $\pm 35 \mathrm{~Hz}$ which gives the reflection heights accurate to about $\pm 1.8, \pm 0.8, \pm 0.6, \pm 0.5$, $\pm 0.4, \pm 0.3 \mathrm{~km}$ for $n=1-6$, respectively, and the electron density to about $\pm 0.7 \mathrm{~cm}^{-3}$ for all harmonics. The increase in $h_{\mathrm{m}}$ with increase in $n$ indicates that the higher order harmonics of same tweeks penetrate deeper into the lower ionosphere while traveling to the receiver by multiple reflections. This supports our consideration that the ionosphere is not sharply bounded at a fixed height for all harmonics but can be considered as sharply bounded (perfectly conducting) progressively at higher altitudes for increasing order of harmonics of tweeks. A difference of about $3.5 \mathrm{~km}$ in $h$ for $n=1-6$ can be established from Fig. 4. Using multimode tweeks up to 8th harmonics and assuming lower ionosphere as collision free, Shvets and Hayakawa (1998) found an increase in $n_{\mathrm{em}}$ from $28-224 \mathrm{~cm}^{-3}$ in the altitude range of about $2 \mathrm{~km}$ at a height of about $81 \mathrm{~km}$. The nighttime $h_{\mathrm{m}}$ reported in this work during a low solar activity period March-December 2006, are higher by 1-2 km than those estimated by Shvets and Hayakawa (1998), indicating a spatial and solar activity dependence of the electron density and hence the reflection heights. The ionospheric VLF reflection height is somewhat lower during solar maximum as compared to solar minimum (McRae and Thomson, 2004). The equivalent electron densities at reflections heights seem to be lower than those reported by Shvets and Hayakawa (1998). Ohya et al. (2003) analyzing the first-order mode cutoff frequency of tweek atmospherics received at Moshiri $\left(44.37^{\circ} \mathrm{N}\right)$ and Kagoshimain $\left(31.48^{\circ} \mathrm{N}\right)$ in Japan, and using the same approach as used by Shvets and Hayakawa (1998), estimated equivalent electron density to vary from $20-28 \mathrm{~cm}^{3}$ at reflection heights of $80-85 \mathrm{~km}$. Solar activity dependence of the $D$-region of the ionosphere is still not clear. Some authors found a decrease in electron density of the lower ionosphere with an increase in the solar activity whereas others found increase in the electron density with an increase in the solar activity (Danilov, 1998 and references therein). 


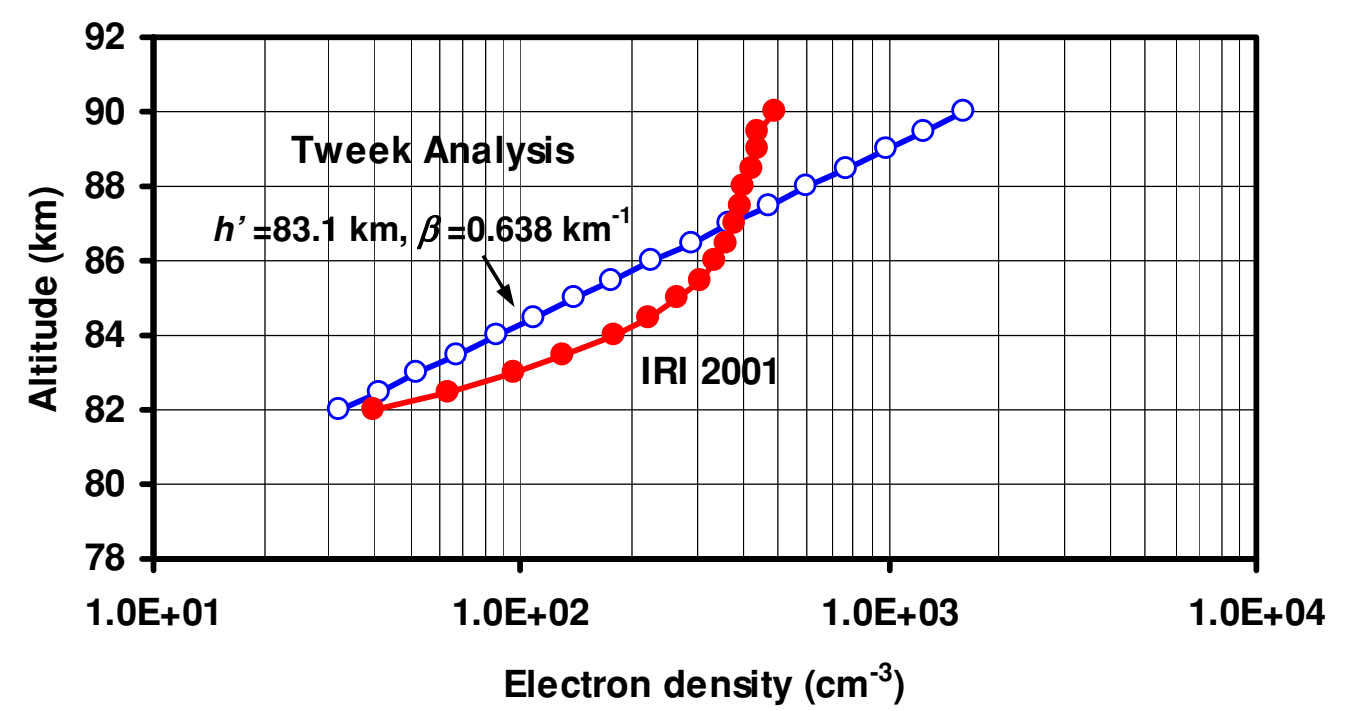

Fig. 5. A comparison of electron density profiles of the lower ionosphere obtained using overall analysis of tweeks (Blue solid line with hollow circles) up to 3rd harmonics and the IRI-2001 model (red solid line with solid circles) at 00:00 hrs LT at location of Suva on 15th day of every month from March-December 2006.

4.3 Equivalent electron density profile and scale height The lower ionosphere can be characterized as the "Wait ionosphere" defined by a reference height $h^{\prime}$ in $\mathrm{km}$ and an exponential sharpness factor $\beta$ in $\mathrm{km}^{-1}$ as considered by many researchers (e.g. Cummer et al., 1998; Cummer and Inan, 2000; Thomson et al., 2007). Lower ionosphere has been studied employing the VLF technique (Cummer et al., 1998) and ELF technique (Cummer and Inan, 2000). Using Long Wave Propagation Code (LWPC), they simulated for a variety of ionospheres, the VLF and ELF spectra of the signals radiated by lightning discharges and received at 1000 $2000 \mathrm{~km}$ away from the lightning source. The value of $\beta$ extracted from the VLF technique by Cummer et al. (1998) was $0.49 \mathrm{~km}^{-1}$ which was lower by as much as $0.15 \mathrm{~km}^{-1}$ than the value of $\beta$ extracted from ELF technique by Cummer and Inan (2000). ELF $(<1.5 \mathrm{kHz})$ radio atmospherics propagate by QTEM mode of wave propagation which can be influenced by $E$ and $F$ regions of the ionosphere (Cummer and Inan, 2000). Comparing the observed nighttime VLF phase and amplitude of navigational transmitters signals with long wave propagation models, Thomson et al. (2007) determined nighttime values of $h^{\prime}=85.1 \pm 0.4 \mathrm{~km}$ and $\beta=0.63 \pm 0.04 \mathrm{~km}^{-1}$ for the mid latitude $D$-region near solar minimum.

Tweek method utilized here gives path-integrated electron density. Substitution of $n_{\mathrm{em}}$ and $h_{\mathrm{m}}$ from Fig. 4 in Eq. (2) for $n=1,2,3$ gives three equations dealing with $h^{\prime}$ and $\beta$ corresponding to the each value of $n$ which on solving yield values of $h^{\prime}$ and $\beta$ as $83.1 \mathrm{~km}$ and $0.638 \mathrm{~km}^{-1}$, respectively. The value of $\beta$ thus obtained is higher by $0.15 \mathrm{~km}^{-1}$ than that obtained by Cummer et al. (1998) using the VLF sferics observed during low solar activity month of July 1996 (Sun spot number $R_{Z} \sim 10$ ) and is consistent with that obtained by Thomson et al. (2007). The period March-December 2006 also falls in lower solar activity period $\left(R_{z} \sim 15\right)$. Figure 5 shows the nighttime $D$-region $n_{\mathrm{e}}$ profile (hollow circle) calculated using Eq. (2) for $h^{\prime}=$ $83.1 \mathrm{~km}$ and $\beta=0.638 \mathrm{~km}^{-1}$ for the altitudes ranges of
$82-90 \mathrm{~km}$. The $n_{\mathrm{em}}$ profile (solid circle) obtained using the IRI-2001 model at 00:00 hrs LT at the location of Suva on 15th day of every month from March-December 2006 is also plotted in Fig. 5. The period March-December 2006 was geomagnetically a quite period and there occurred no geomagnetic storms on 14th or 15th days of the months up to November. However, a Sudden Storm Commencement occurred at 14:14 UT on 14 December that caused an intense geomagnetic storm with a minimum $D_{\text {st }}$ index (on line at http://swdcwww.kugi.kyoto-u.ac.jp) value of $-146 \mathrm{nT}$ at 08:00 UT (20:00 LT) on 15 December. Such storms may increase lower ionospheric electron density and hence decrease the reflection heights (Ohya et al., 2006). Unfortunately our recoding of ELF-VLF data was off during 1317 December 2006 due to some technical problem with the recording system. Thus $n_{\mathrm{em}}$ values obtained using the IRI2001 model at 00:00 hrs LT on 15th day of every month can be considered as good estimates of nighttime monthly mean values at our location (Suva). The values of $n_{\mathrm{em}}$ obtained by tweek analysis are lower by $20-45 \%$ at the altitudes $82-86 \mathrm{~km}$ with a very good match at about $87 \mathrm{~km}$. The trend of variation of the $n_{\mathrm{em}}$ in the lower ionosphere around the altitude $82-86 \mathrm{~km}$ is almost the same, indicating that tweek method is useful for estimating the electron density profile of the lower part of the nighttime $D$-region ionosphere. Tweek method corresponds to a scale height of $1.9 \mathrm{~km}$ (Fig. 5) in terms of usually used exponential electron density profile whereas the scale height calculated using the IRI-2001 values of $n_{\mathrm{em}}$ is about $1.5 \mathrm{~km}$. The characteristic scale height estimated using the tweeks is consistent with the results $(1.6 \mathrm{~km})$ of Hughes et al. (1974) but higher in comparison to that estimated $(1 \mathrm{~km})$ by Shvets and Hayakawa (1998) from the measurement of night-time ELF-VLF sferics spectra.

\section{Summary}

The nighttime VLF reflection heights from the cut-off frequency of different harmonics of tweeks atmospherics 
at a low latitude station in Fiji were obtained using waveguide mode theory. An expression for electron density at the VLF ionospheric reflection heights is obtained using magnetoionic theory of wave propagation in the ionosphere which is weakly ionized inhomogeneous, anisotropic and collisional plasma. Equivalent electron densities ranged from $29-170 \mathrm{~cm}^{-3}$ in the altitude range of about $3.5 \mathrm{~km}$ at the height of $82.8 \mathrm{~km}$. The main focus of this work is to obtain the electron density profile of the lower ionosphere using cutoff frequency of first three harmonics which has not been attempted so far using tweek measurements. From the analysis of the first three harmonics of tweeks the values of $h^{\prime}$ and $\beta$ are calculated to be $83.1 \mathrm{~km}$ and $0.64 \mathrm{~km}^{-1}$, respectively. Equivalent electron density profile of the nighttime lower ionosphere shows lower values then those obtained from the IRI-2001 model, however, the trend of electron density variation in the lower ionosphere in the altitude range of $82-86 \mathrm{~km}$ is almost the same. Nighttime $D$-region scale height is estimated to be $1.9 \mathrm{~km}$.

Acknowledgments. This work was supported by the University Research Committee (URC) under the URC grant no. 6384. Authors thank director of WWLLN for proving VLF receiving system and global lightning location data.

\section{References}

Budden, K. G., Radio Waves in the ionosphere, Cambridge University Press, New York, 1961.

Cummer, S. A. and U. S. Inan, Ionospheric $E$ region remote sensing with ELF radio atmospherics, Radio Sci., 35, 1437-1444, 2000.

Cummer, S. A., U. S. Inan, and T. F. Bell, Ionospheric $D$ region remote sensing using VLF radio atmospherics, Radio Sci., 33, 1781-1792, 1998.

Danilov, A. D., Solar cycle variations of the D-region ionosphere, Ann. Geophys., 25, 1527-1533, 1998.

Dowden, R. L., J. B. Bruendell, and C. J. Rodger, VLF lightning location by time of group arrival (TOGA) at multiple sites, J. Atmos. Sol.-Terr. Phys., 64, 817-830, 2002.
Ferencz, O. E., Cs. Ferencz, P. Steinbach, J. Lichtenberger, D. Hamar, M. Parrot, F. Lefeuvre, and J. J. Berthelier, The effects of subionospheric propagation on whistlers recorded by the DEMETER satelliteobservation and modeling, Ann. Geophys., 25, 1103-1112, 2007.

Hayakawa, M., K. Ohta, and K. Baba, Wave characteristics of tweek atmospherics deduced from the direction-finding measurement and theoretical interpretation, J. Geophys. Res., 99, 10,733-10,743, 1994.

Hughes, H. G., R. J. Gallenburger, and R. A. Pappert, Evaluation of nighttime exponential ionospheric models using VLF atmospherics, Radio Sci., 9, 1109-1116, 1974.

Kumar, S., A. Kishore, and V. Ramachandran, Higher harmonic tweek sferics observed at low latitude: Estimation of VLF reflection heights and tweeks propagation distance, Ann. Geophys., 25, 1451-1459, 2008.

McRae, W. M. and N. R. Thomson, Solar flare induced ionospheric $D$ region enhancements from VLF phase and amplitude observations, $J$. Atmos. Sol.-Terr. Phys., 66, 77-87, 2004.

Ohya, H., M. Nishino, Y. Murayama, and K. Igarashi, Equivalent electron densities at reflection heights of tweek atmospherics in the low-middle latitude D-region ionosphere, Earth Planets Space, 55, 627-635, 2003.

Ohya, H., M. Nishino, Y. Murayama, K. Igarashi, and A. Saito, Using tweek atmospherics to measure the response of the low-middle latitude D-region ionosphere to a magnetic storm, J. Atmos. Sol.-Terr. Phys., 68, 697-709, 2006.

Ohya, H., K. Shiokawa, and Y. Miyoshi, Development of an automatic procedure to estimate the reflection height of tweek atmospherics, Earth Planets Space, 60, 837-843, 2008.

Ratcliffe, J. A., The magneto-ionic theory and its applications to the ionosphere, Cambridge University Press, Cambridge, 1959.

Rodger, C. J., S. W. Werner, J. B. Brundell, N. R. Thomson, E. H. Lay, R. H. Holzworth, and R. L. Dowden, Detection efficiency of the VLF World-Wide Lightning Location Network (WWLLN): Initial case study, Ann. Geophys., 24, 3197-3214, 2006.

Shvets, A. V. and M. Hayakawa, Polarization effects for tweek propagation, J. Atmos. Sol.-Terr. Phys., 60, 461-469,1998.

Thomson, N. R., M. A. Clilverd, and W. M. McRae, Nighttime $D$ region parameters from VLF amplitude and Phase, J. Geophys. Res., 112, A07304, doi:10.1029/2007JA01227, 2007.

Wait, J. R. and K. P. Spies, Characteristics of the Earth-ionosphere waveguide for VLF radio waves, NBS Tech. Not., 300 pp., 1964.

Yamashita, M., Propagation of tweek atmospherics, J. Atmos. Terr. Phys., 40, 151-156, 1978.

S. Kumar (e-mail: kumar_su@usp.ac.fj), A. Deo, and V. Ramachandran 